\title{
A RIGHT INVERSE OF THE ASKEY-WILSON OPERATOR
}

\author{
B. MALCOLM BROWN AND MOURAD E. H. ISMAIL
}

(Communicated by Hal Smith)

\begin{abstract}
We establish an integral representation of a right inverse of the Askey-Wilson finite difference operator on $L^{2}$ with weight $\left(1-x^{2}\right)^{-1 / 2}$. The kernel of this integral operator is $\vartheta_{4}^{\prime} / \vartheta_{4}$ and is the Riemann mapping function that maps the interior of an ellipse conformally onto the open unit disc.
\end{abstract}

\section{INTRODUCTION}

In 1985 Askey and Wilson introduced what has become known as the AskeyWilson operator. It is defined in the following way. Given a function $f(x)$ with $x=\cos \theta$, then $f(x)$ can be viewed as a function of $e^{i \theta}$. Let

$$
\check{f}\left(e^{i \theta}\right):=f(x), \quad x=\cos \theta .
$$

In this notation the Askey-Wilson finite difference operator $\mathscr{D}_{q}$ is defined by

$$
\left(\mathscr{D}_{q} f\right)(x):=\frac{\left(\delta_{q} \check{f}\right)\left(e^{i \theta}\right)}{\left(\delta_{q} \check{h}\right)\left(e^{i \theta}\right)},
$$

where $h(x)=x$ and

$$
\left(\delta_{q} g\right)\left(e^{i \theta}\right)=g\left(q^{1 / 2} e^{i \theta}\right)-g\left(q^{-1 / 2} e^{i \theta}\right) .
$$

It follows easily from equality $(1.3)$ that $\breve{h}(z)=\frac{1}{2}\left(z+z^{-1}\right)$ and $\delta_{q} \breve{h}(x)=$ $\left(q^{1 / 2}-q^{-1 / 2}\right) i \sin \theta$. Thus

$$
\left(\mathscr{D}_{q} f\right)(x)=\frac{\check{f}\left(q^{1 / 2} e^{i \theta}\right)-\check{f}\left(q^{-1 / 2} e^{i \theta}\right)}{i\left(q^{1 / 2}-q^{-1 / 2}\right) \sin \theta} .
$$

The operator $\mathscr{D}_{q}$ was introduced in [2], and in the theory of the AskeyWilson polynomials it plays an analogous role to that of differentiation in the theory of Jacobi, Hermite, and Laguerre polynomials. Alphonse Magnus [7] provided a general setting for finite difference operators and indicated how the Askey-Wilson operator arises in a very natural way as a finite difference operator.

Received by the editors September 28, 1993.

1991 Mathematics Subject Classification. Primary 33D45, 42C10; Secondary 45E10.

Key words and phrases. Integral operator, Chebyshev polynomials, theta functions, finite difference operators, conformal mappings, $q$-Hermite polynomials.

Research partially supported by NSF grant DMS 9203659, NATO grant CRG 931216, and a grant from SERC. 
We remark that $\mathscr{D}_{q}$ remains invariant if $q$ is replaced by $1 / q$. In this work we will avoid $q$ 's on the unit circle, thus there is no loss of generality in assuming $|q|<1$.

The Chebyshev polynomials $T_{n}$ and $U_{n}$ of the first and second kinds, respectively, are

$$
T_{n}(x)=\cos n \theta, \quad U_{n}(x)=\frac{\sin ((n+1) \theta)}{\sin \theta}, \quad x=\cos \theta,
$$

and are orthogonal on $[-1,1]$ with respect to the weight functions $\left(1-x^{2}\right)^{-1 / 2}$ and $\left(1-x^{2}\right)^{1 / 2}$, respectively. A calculation gives

$$
\mathscr{D}_{q} T_{n}(x)=\frac{q^{n / 2}-q^{-n / 2}}{q^{1 / 2}-q^{-1 / 2}} U_{n-1}(x) .
$$

The Askey-Wilson polynomials $p_{n}(x ; a, b, c, d \mid q)$ [2], [3], which depend on four parameters, are orthogonal on $[-1,1]$ when all their parameter values are in $(-1,1)$. Thus one would like to study the action of $\mathscr{D}_{q}$ on functions defined on $[-1,1]$. One difficulty in defining the action of $\mathscr{D}_{q}$, as defined in (1.4), is that (1.4) uses values of $f$ outside $[-1,1]$. In fact the definition (1.4) given in [2] uses values of $f$ at points in the complex plane without specifying how $f$ is extended to the whole, or part of, the $x$-plane. To rectify these difficulties we propose to define $\mathscr{D}_{q}$ on a dense subset of $L^{2}\left[\left(1-x^{2}\right)^{-1 / 2},[-1,1]\right]$. We propose the following definition.

Definition 1.1. Let $f \in L^{2}\left[\left(1-x^{2}\right)^{-1 / 2},[-1,1]\right]$, and assume that $f$ has a Fourier-Chebyshev expansion

$$
f(x) \sim \sum_{n=0}^{\infty} f_{n} T_{n}(x),
$$

with the Fourier-Chebyshev coefficients $\left\{f_{n}\right\}$ satisfying

$$
\sum_{n=0}^{\infty}\left|\left(1-q^{n}\right) q^{-n / 2} f_{n}\right|^{2}<\infty
$$

We then define $\mathscr{D}_{q} f$ as the unique (almost everywhere) function whose FourierChebyshev expansion is

$$
\left(\mathscr{D}_{q} f\right)(x) \sim \sum_{n=0}^{\infty} \frac{q^{n / 2}-q^{-n / 2}}{q^{1 / 2}-q^{-1 / 2}} f_{n} U_{n-1}(x) .
$$

Such functions $f$ we call $q$-differentiable.

Clearly, (1.7) is satisfied on a dense subset $S$ of $L^{2}\left[\left(1-x^{2}\right)^{-1 / 2},[-1,1]\right]$. It is also clear that $\mathscr{D}_{q}$ maps $S$ into a dense subset of $L^{2}\left[\left(1-x^{2}\right)^{1 / 2},[-1,1]\right]$.

In Section 2 we define a right inverse to $\mathscr{D}_{q}$, which will be denoted as $\mathscr{D}_{q}^{-1}$, and find an integral representation for this inverse operator. We shall show that the operator $\mathscr{D}_{q}^{-1}$ is a convolution operator whose kernel is $\vartheta_{4}^{\prime} / \vartheta_{4}$. Also in Section 2 we prove that $\mathscr{D}_{q} \mathscr{D}_{q}^{-1}$ is the identity operator on $L^{2}\left[\left(1-x^{2}\right)^{-1 / 2},[-1,1]\right]$. Further we prove that if $g(x)$ is a piecewise continuous function on $[-1,1]$ with $\mathscr{D}_{q}$ defined by $(1.4)$, then $\left(\mathscr{D}_{p} \mathscr{D}_{q}^{-1} g\right)(x)$ 
converges to $g(x)$ as $p \rightarrow q^{+}$at the points of continuity of $g$. Section 3 contains remarks on related work and establishes a connection between the kernel of $\mathscr{D}_{q}^{-1}$ and the Riemann mapping function of the interior of an ellipse to the open unit disc. The definition (1.9) of $\mathscr{D}_{q}$ uses $\left\{T_{n}(x)\right\}$ as a basis for a function space. In Section 4 we use a different basis, namely the Rogers $q$-Hermite polynomials, in the $L^{2}$ spaces weighted by their weight function. We again identify $\mathscr{D}_{q}^{-1}$ as an integral operator and find its kernel explicitly.

\section{AN INTEGRAL OPERATOR}

We seek an operator $\mathscr{D}_{q}^{-1}$ so that

$$
\mathscr{D}_{q} \mathscr{D}_{q}^{-1}=I .
$$

Let $\mathscr{D}_{q} f=g$ so that

$$
f(x) \sim \sum_{n=0}^{\infty} f_{n} T_{n}(x), \quad g(x) \sim \sum_{n=1}^{\infty} g_{n} U_{n-1}(x),
$$

and

$$
f_{n}=g_{n} \frac{\left(q^{1 / 2}-q^{-1 / 2}\right)}{\left(q^{n / 2}-q^{-n / 2}\right)}, \quad n>0 .
$$

We first use a heuristic (or formal) approach to find a way to recover $f$ from the knowledge of $g$. Formally we have

$$
\begin{aligned}
\sum_{n=1}^{\infty} f_{n} T_{n}(x) & =\sum_{n=1}^{\infty} g_{n} \frac{\left(q^{1 / 2}-q^{-1 / 2}\right)}{\left(q^{n / 2}-q^{-n / 2}\right)} T_{n}(x) \\
& =\frac{2}{\pi} \sum_{n=1}^{\infty}\left(\int_{-1}^{1} g(y) U_{n-1}(y) \sqrt{1-y^{2}} d y\right) \frac{\left(q^{1 / 2}-q^{-1 / 2}\right)}{\left(q^{n / 2}-q^{-n / 2}\right)} T_{n}(x) \\
& =\frac{2}{\pi}(1-q) q^{-1 / 2} \int_{-1}^{1} g(y)\left[\sum_{n=1}^{\infty} \frac{T_{n}(x) U_{n-1}(y)}{1-q^{n}} q^{n / 2}\right] \sqrt{1-y^{2}} d y .
\end{aligned}
$$

This suggests defining $\mathscr{D}_{q}^{-1}$ as an integral operator whose kernel is

$$
F(x, y):=\frac{2(1-q)}{\pi \sqrt{q}} \sum_{n=1}^{\infty} \frac{T_{n}(x) U_{n-1}(y)}{1-q^{n}} q^{n / 2} .
$$

Set

$$
x=\cos \theta, \quad y=\cos \phi
$$

to get

$$
\begin{aligned}
& F(\cos \theta, \cos \phi)=\frac{(1-q) q^{-1 / 2}}{\pi \sin \phi} \sum_{n=1}^{\infty} \frac{2 \cos (n \theta) \sin (n \phi)}{1-q^{n}} q^{n / 2} \\
& =\frac{(1-q) q^{-1 / 2}}{\pi \sin \phi} \sum_{n=1}^{\infty} \frac{q^{n / 2}}{1-q^{n}}[\sin (n(\theta+\phi))-\sin (n(\theta-\phi))],
\end{aligned}
$$


and observe that

$$
\begin{aligned}
\int_{-1}^{1} F(x, y) g(y) \sqrt{1-y^{2}} d y & =\int_{0}^{\pi} F(\cos \theta, \cos \phi) g(\cos \phi) \sin ^{2} \phi d \phi \\
& =\int_{-\pi}^{\pi} G(\cos \theta, \cos \phi) g(\cos \phi) \sin \phi d \phi
\end{aligned}
$$

where

$$
G(\cos \theta, \cos \phi)=\frac{(1-q)}{\pi \sqrt{q}} \sum_{n=1}^{\infty} \frac{q^{n / 2}}{1-q^{n}} \sin (n(\theta+\phi)) .
$$

We next recall that the theta function $\vartheta_{4}(z, q)$ may be defined by

$$
\vartheta_{4}(z, q)=\sum_{-\infty}^{\infty}(-1)^{n} q^{n^{2}} e^{2 i n z}
$$

(see [11, p. 463]), the logarithmic derivative of which has the Fourier series expansion

$$
\frac{\vartheta_{4}^{\prime}(z, q)}{\vartheta_{4}(z, q)}=4 \sum_{n=1}^{\infty} \frac{q^{n}}{1-q^{2 n}} \sin (2 n z)
$$

[11, Example 11, p. 489]. Thus (2.3), (2.4), (2.5), and (2.6) motivate the following definition:

Definition 2.1. The operator $\mathscr{D}_{q}^{-1}$ is defined on $L^{2}\left[\left(1-x^{2}\right)^{1 / 2},[-1,1]\right]$ as the integral operator

$$
\left(\mathscr{D}_{q}^{-1} g\right)(\cos \theta)=\frac{1-q}{4 \pi \sqrt{q}} \int_{-\pi}^{\pi} \frac{\vartheta_{4}^{\prime}((\theta-\phi) / 2, \sqrt{q})}{\vartheta_{4}((\theta-\phi) / 2, \sqrt{q})} g(\cos \phi) \sin \phi d \phi .
$$

Observe that the kernel of the integral operator (2.9) is bounded when $(x, y)$ $(=(\cos \theta, \cos \phi)) \in[-1,1] \times[-1,1]$. Thus the operator $\mathscr{D}_{q}^{-1}$ is well defined and bounded on $L^{2}\left[\left(1-x^{2}\right)^{1 / 2},[-1,1]\right]$. Furthermore $\mathscr{D}_{q}^{-1}$ is a one-to-one mapping from $L^{2}\left[\left(1-x^{2}\right)^{1 / 2},[-1,1]\right]$ into $L^{2}\left[\left(1-x^{2}\right)^{-1 / 2},[-1,1]\right]$.

Theorem 2.1. The operator $\mathscr{D}_{q} \mathscr{D}_{q}^{-1}$ is the identity operator on

$$
L^{2}\left[\left(1-x^{2}\right)^{1 / 2},[-1,1]\right]
$$

Proof. Replace $\vartheta_{4}^{\prime} / \vartheta_{4}$ in (2.9) by the expansion (2.8); then apply Parseval's formula. In view of the uniform convergence of the series in (2.8) we may reverse the order of integration and summation in (2.9) from which it follows that the steps leading to Definition 2.1 can now be reversed, yielding the result.

Note that the kernel $G$ of $(2.6)$ is defined and bounded for all $\phi \in[-\pi, \pi]$ and all $\theta$ for which $\left|q^{1 / 2} e^{i \theta}\right| \leq 1$, where we have written $e^{i \theta}:=x+\sqrt{x^{2}-1}$ and the branch of the square root is chosen so that $\sqrt{x^{2}-1} \approx x$ as $x \rightarrow \infty$. This convention makes $\left|e^{-i \theta}\right| \leq\left|e^{i \theta}\right|$ with equality if and only if $\theta$ is real, that is $x \in[-1,1]$. This extends the definition of $\mathscr{D}_{q}^{-1}$ to the interior of the ellipse $\left|z+\sqrt{z^{2}-1}\right|=q^{1 / 2}$ in the complex $z$-plane. This ellipse has foci at 
\pm 1 , and its major and minor axes are $q^{-1 / 2} \pm q^{1 / 2}$, respectively. Its equation in the $x y$-plane is

$$
\frac{x^{2}}{a^{2}}+\frac{y^{2}}{b^{2}}=1, \quad a=\frac{1}{2}\left(q^{-1 / 2}+q^{1 / 2}\right), \quad b=\frac{1}{2}\left(q^{-1 / 2}-q^{1 / 2}\right) .
$$

It is worth mentioning that provided we exercise some care the Askey-Wilson definition (1.4) yields the result $\mathscr{D}_{q} \mathscr{D}_{q}^{-1}=I$. One reason for being particularly careful is that $\mathscr{D}_{q}^{-1} g$ may not be in the domain of $\mathscr{D}_{q}$ because in order to use (1.3) we need to assume that $f$ has an analytic extension to a domain in the complex plane containing $\left|z \pm \sqrt{z^{2}-1}\right| \leq q^{-1 / 2}$. Let $f(\cos \theta)$ denote the right-hand side of (2.9). From the discussion following Theorem 2.1 it is easy to see that $\mathscr{D}_{p} f(x)$ is well defined provided that $1>p>q$. Indeed we find

$$
\begin{aligned}
\left(\mathscr{D}_{p} f\right)(\cos \theta)= & \int_{-\pi}^{\pi} \mathscr{D}_{p} G(\cos \theta, \cos \phi) g(\cos \phi) \sin \phi d \phi \\
= & \frac{(1-q) p^{1 / 2}}{\pi(1-p) q^{1 / 2} \sin \theta} \\
& \times \int_{-\pi}^{\pi} \sum_{n=1}^{\infty} \frac{\left(1-p^{n}\right)}{\left(1-q^{n}\right)}(q / p)^{n / 2} \cos (n(\theta+\phi)) g(\cos \phi) \sin \phi d \phi .
\end{aligned}
$$

By writing $\frac{1-p^{n}}{1-q^{n}}$ as $1+\frac{q^{n}-p^{n}}{1-q^{n}}$ and denoting $q / p$ by $r^{2}$ we see that replacing $\phi$ by $-\phi$ gives

$$
\begin{aligned}
\lim _{p \rightarrow q^{+}}\left(\mathscr{D}_{p} \mathscr{D}_{q}^{-1} g\right)(\cos \theta)= & \frac{1}{\pi \sin \theta} \lim _{r \rightarrow 1^{-}} \int_{-\pi}^{\pi}\left[\sum_{n=1}^{\infty} r^{n} \cos (n(\theta-\phi))\right] \\
\times & g(\cos \phi) \sin \phi d \phi+0 \\
= & \frac{1}{\pi \sin \theta} \lim _{r \rightarrow 1^{-}} \int_{-\pi}^{\pi}\left[\frac{1}{2}+\sum_{n=1}^{\infty} r^{n} \cos (n(\theta-\phi))\right] \\
\times & \quad g(\cos \phi) \sin \phi d \phi,
\end{aligned}
$$

since $g(\cos \phi) \sin \phi$ is an odd function. Therefore

$$
\lim _{p \rightarrow q^{+}}\left(\mathscr{D}_{p} \mathscr{D}_{q}^{-1} g\right)(\cos \theta)=\lim _{r \rightarrow 1^{-}} \frac{1}{2 \pi \sin \theta} \int_{-\pi}^{\pi} \frac{\left(1-r^{2}\right) g(\cos \phi) \sin \phi}{1-2 r \cos (\theta-\phi)+r^{2}} d \phi .
$$

Finally the above limit exists and equals $g(\cos \theta)$ at the points of continuity of $g$ if $g(\cos \theta)$ is continuous on $[-\pi, \pi]$ except for finitely many jumps $[9, \mathrm{p}$. 147]. Thus we have proved the following result.

Theorem 2.2. Let $g(x)$ be a continuous function on $[-1,1]$ except for finitely many jumps. Then with $\mathscr{D}_{q}$ defined as in (1.4), the limiting relation

$$
\lim _{p \rightarrow q^{+}}\left(\mathscr{D}_{p} \mathscr{D}_{q}^{-1} g\right)(x)=g(x)
$$

holds at the points of continuity of $g$. 


\section{REMARKS}

The kernel $\vartheta_{4}^{\prime} / \vartheta_{4}$ of $(2.8)$ has appeared earlier in conformal mappings. Let $\zeta$ be a fixed point in the interior of the ellipse (2.10) in the complex plane, and let $f(z, \zeta)$ be the function that maps the interior of the ellipse (2.10) conformally onto the open unit disc and satisfies $f(\zeta, \zeta)=0$ and $f^{\prime}(\zeta, \zeta)>0$. It is known $[8$, p. 260$]$, that

$$
f(z, \zeta)=g(z, \zeta)-g(\zeta, \zeta)
$$

where

$$
g(z, \zeta)=\sqrt{\frac{\pi}{K(\zeta, \zeta)}} \sum_{n=1}^{\infty} \frac{T_{n}(z) \overline{U_{n-1}(\zeta)}}{\rho^{n}-\rho^{-n}}
$$

and the Bergman kernel $K(z, \zeta)$ is

$$
K(z, \zeta):=\frac{4}{\pi} \sum_{n=0}^{\infty} \frac{(n+1) U_{n}(z) \overline{U_{n}(\zeta)}}{\rho^{n+1}-\rho^{-n-1}}, \quad \rho:=(a+b)^{2}=\left(b+\sqrt{b^{2}+1}\right)^{2} .
$$

In fact the Bergman kernel of the ellipse is a constant multiple of $f^{\prime}(z, \zeta)$. It is clear that $g(z, \zeta)$ is a constant multiple of our kernel $G(2.6)$ with $\rho=q^{-1 / 2}$, so that $q=\left(b+\sqrt{\left.b^{2}+1\right)}\right)^{-4}=e^{-4 u}$ if $b=\sinh u$.

The connection between the Riemann mapping function $f(z, \zeta)$ of the ellipse (2.10) and our kernel may seem very surprising at a first glance. However this may not be a complete surprise because if $f$ is real analytic in $(-1,1)$, it will have an extension which is analytic in the open unit disc and (1.4) will be meaningful if $\left|q^{1 / 2} e^{i \theta}\right|<1$, which is the interior of the ellipse (2.10). Furthermore the Chebyshev polynomials $\left\{U_{n}(z)\right\}$ are orthogonal on the unit disc with respect to the Lebesgue measure in the plane.

After this work was completed Ismail and Zhang [5] proved that the eigenvalues of the integral operator (2.9) are $\pm i / j_{0, k}(q)$ where $\left\{j_{\nu, k}(q)\right\}$ are the zeros of the $q$-Bessel function $J_{\nu}^{(2)}(z ; q)$ (see [4] or [3] for the definition of $\left.J_{\nu}^{(2)}(z ; q)\right)$. Ismail and Zhang also proved that the eigenfunctions of $\mathscr{D}_{q}^{-1}$ provide a new $q$-exponential function. In addition they found the eigenvalues and eigenfunctions of $\mathscr{D}_{q}^{-1}$ on $L^{2}$ spaces weighted by the weight functions of the Jacobi and continuous $q$-ultraspherical polynomials [1], [2]. Later Ismail, Rahman, and Zhang [6] extended this investigation to the weights of continuous $q$-Jacobi polynomials [3].

\section{THE $q$-HeRMITE SPACE}

It is a consequence of our definition of $\mathscr{D}_{q}$, as in (1.7)-(1.9), that that subset $S$ of $\left.L^{2}\left[\left(1-x^{2}\right)^{-1 / 2}\right],[-1,1]\right]$ of $q$-differentiable functions gets mapped into $\left.L^{2}\left[\left(1-x^{2}\right)^{1 / 2}\right],[-1,1]\right]$. In this section we shall define $\mathscr{D}_{q}$ on a different weighed $L^{2}$ space in such a way that $q$-differentiable functions are mapped into the same space. 
Recall the definition of the $q$-shifted factorial [3],

(4.1) $(a ; q)_{0}:=1, \quad(a ; q)_{n}:=\prod_{j=1}^{n}\left(1-a q^{j-1}\right), \quad n=1,2, \ldots$ or $\infty$,

and the multiparameter notation

$$
\left(a_{1}, \ldots, a_{m} ; q\right)_{n}=\prod_{k=1}^{m}\left(a_{k} ; q\right)_{n} .
$$

The $q$-Hermite polynomials of L. J. Rogers are [1], [3]

$$
H_{n}(x \mid q):=\sum_{k=0}^{n} \frac{(q ; q)_{n}}{(q ; q)_{k}(q ; q)_{n-k}} e^{i(n-2 k) \theta}, \quad x=\cos \theta .
$$

The orthogonality relation of the $H_{n}$ 's is [1], [3]

$$
\int_{-1}^{1} H_{m}(x \mid q) H_{n}(x \mid q) w(x) d x=\frac{2 \pi(q ; q)_{n}}{(q ; q)_{\infty}} \delta_{m, n},
$$

where the weight function $w$ is

$$
w(x)=\left(1-x^{2}\right)^{-1 / 2} \prod_{n=0}^{\infty}\left(1-2\left(2 x^{2}-1\right) q^{n}+q^{2 n}\right),
$$

that is, $w(\cos \theta)=\left(e^{2 i \theta}, e^{-2 i \theta} ; q\right)_{\infty} / \sin \theta$. The $H_{n}$ 's have the generating function

$$
\sum_{n=0}^{\infty} \frac{H_{n}(x \mid q)}{(q ; q)_{n}} r^{n}=1 /\left(r e^{i \theta}, r e^{-i \theta} ; q\right)_{\infty}
$$

By applying $\mathscr{D}_{q}$ to both sides of (4.6) we get

$$
\mathscr{D}_{q} H_{n}(x \mid q)=2 q^{(1-n) / 2} \frac{\left(1-q^{n}\right)}{(1-q)} H_{n-1}(x \mid q) .
$$

One can then follow the procedure of Section 2 except that we now work with the function space $L^{2}[w(x),[-1,1]]$ and $\mathscr{D}_{q}$ maps it into itself. We then establish, in a straightforward manner, the integral representation

$$
\left(\mathscr{D}_{q}^{-1} g\right)(x)=\frac{(1-q)(q ; q)_{\infty}}{4 \pi \sqrt{q}} \int_{-1}^{1} H(x, t, \sqrt{q}) g(t) w(t) d t,
$$

where the more general kernel $H(x, t, r)$ is defined to be

$$
H(x, t, r)=\sum_{n=1}^{\infty} \frac{r^{n}}{(q ; q)_{n}} H_{n}(x \mid q) H_{n-1}(t \mid q) .
$$

We now evaluate the kernel $H(x, t, r)$. 
Theorem 4.1. We have

$$
\begin{aligned}
H(\cos \theta & , \cos \phi, r) \\
= & \frac{-2 r\left(q r^{2} ; q\right)_{\infty}}{\left(r e^{i(\theta+\phi)}, r e^{i(\theta-\phi)}, r e^{-i(\theta+\phi)}, r e^{-i(\theta-\phi)} ; q\right)_{\infty}} \\
& \times \sum_{n=0}^{\infty} \frac{\left(r e^{i(\theta+\phi)}, r e^{i(\theta-\phi)}, r e^{-i(\theta+\phi)}, r e^{-i(\theta-\phi)} ; q\right)_{n}}{\left(q r^{2} ; q\right)_{2 n}} \\
& \times\left[-\cos \theta+r q^{n} \cos \phi\right] q^{n} .
\end{aligned}
$$

Proof. The Poisson kernel of the Rogers $q$-Hermite polynomials is

$$
\begin{aligned}
\sum_{n=0}^{\infty} & \frac{H_{n}(\cos \theta \mid q) H_{n}(\cos \phi \mid q)}{(q ; q)_{n}} r^{n} \\
& =\frac{\left(r^{2} ; q\right)_{\infty}}{\left(r e^{i(\theta+\phi)}, r e^{i(\theta-\phi)}, r e^{-i(\theta+\phi)}, r e^{-i(\theta-\phi)} ; q\right)_{\infty}}
\end{aligned}
$$

Keeping $\theta$ fixed and applying $\mathscr{D}_{q}$, acting on the variable $\cos \phi$, to (4.11) give

$$
\begin{aligned}
\sum_{n=1}^{\infty} & \frac{H_{n}(\cos \theta \mid q) H_{n-1}(\cos \phi \mid q)}{(q ; q)_{n}} r^{n} q^{-n / 2}\left(1-q^{n}\right) \\
& =\frac{-2\left(r^{2} ; q\right)_{\infty} r q^{-1 / 2}\left(-\cos \theta+r q^{-1 / 2} \cos \phi\right)}{\left(r q^{-1 / 2} e^{i(\theta+\phi)}, r q^{-1 / 2} e^{i(\theta-\phi)}, r q^{-1 / 2} e^{-i(\theta+\phi)}, r q^{-1 / 2} e^{-i(\theta-\phi)} ; q\right)_{\infty}}
\end{aligned}
$$

This implies

$$
\begin{aligned}
& H\left(\cos \theta, \cos \phi, r q^{-1 / 2}\right)-H\left(\cos \theta, \cos \phi, r q^{1 / 2}\right) \\
& \quad=\frac{-2\left(r^{2} ; q\right)_{\infty} r q^{-1 / 2}\left(-\cos \theta+r q^{-1 / 2} \cos \phi\right)}{\left(r q^{-1 / 2} e^{i(\theta+\phi)}, r q^{-1 / 2} e^{i(\theta-\phi)}, r q^{-1 / 2} e^{-i(\theta+\phi)}, r q^{-1 / 2} e^{-i(\theta-\phi)} ; q\right)_{\infty}} .
\end{aligned}
$$

Next replace $r$ by $r q^{k+1 / 2}$ and add the resulting equations for $k=0,1, \ldots$. In view of $H(x, y, 0)=0$ the result is

$$
H(x, y, r)=\sum_{k=0}^{\infty} \frac{r q^{k}\left(-\cos \theta+t q^{k} \cos \phi\right)\left(r^{2} q^{2 k+1} ; q\right)_{\infty}}{\left(r q^{k} e^{i(\theta+\phi)}, r q^{k} e^{i(\theta-\phi)}, r q^{k} e^{-i(\theta+\phi)}, r q^{k} e^{-i(\theta-\phi)} ; q\right)_{\infty}}
$$

The above equation is equivalent to (4.10), and the proof is complete.

Note that the right-hand side of $(4.10)$ can be written as a sum of two ${ }_{5} \phi_{4}$ functions.

\section{ACKNOWLEDGMENTS}

Thanks to Richard Askey for comments and encouragement and to Ruiming Zhang for reference [8]. Thanks also to the referee for pointing out several misprints in the original version. 


\section{REFERENCES}

1. R. Askey and M. E. H. Ismail, A generalization of ultraspherical polynomials, Studies in Pure Mathematics (P. Erdös, ed.), Birkhauser, Basel, 1983, pp. 55-78.

2. R. Askey and J. Wilson, Some basic hypergeometric polynomials that generalize Jacobi polynomials, Mem. Amer. Math. Soc., Number 319, Amer. Math. Soc., Providence, RI, 1985.

3. G. Gasper and M. Rahman, Basic hypergeometric series, Cambridge Univ. Press, Cambridge, 1990.

4. M. E. H. Ismail, The zeros of basic Bessel functions, the functions $J_{v+a x}(x)$, and associated orthogonal polynomials, J. Math. Anal. Appl. 82 (1982), 1-19.

5. M. E. H. Ismail and R. Zhang, Diagonalization of certain integral operators, Adv. Math. 109 (1994), 1-33.

6. M. E. H. Ismail, M. Rahman, and R. Zhang, Diagonalization of certain integral operators II, J. Comput. Appl. Math. (to appear).

7. A. P. Magnus, Associated Askey-Wilson polynomials as Laguerre-Hahn orthogonal polynomials, Orthogonal Polynomials and Their Applications (M. Alfaro et al., eds.), Lecture Notes in Math., vol. 1329, Springer-Verlag, Berlin, 1988, pp. 261-278.

8. Z. Nehari, Conformal mapping, McGraw-Hill, New York, 1952.

9. __ Introduction to complex analysis, Allyn and Bacon, Boston, MA, 1961.

10. G. Szegö, Orthogonal polynomials, fourth edition, Amer. Math. Soc., Providence, RI, 1975.

11. E. T. Whittaker and G. N. Watson, A course of modern analysis, second edition, Cambridge Univ. Press, Cambridge, 1927.

Department of Computing Mathematics, University of Wales College of Cardiff, Mathematics Institute, Senghennydd Road, Cardiff CF2 4YN, United Kingdom

E-mail address: malcolm@cm.cf.ac.uk

Department of Mathematics, University of South Florida, Tampa, Florida 33620

E-mail address: ismail@hahn.math.usf.edu 